\title{
Post Treatment Process and Selective Laser Sintering Mechanism of Polymer-Coated Mo Powder
}

\author{
Bin Liu*, Peikang Bai and Yuxin Li \\ School of Material Science and Engineering, North University of China, Taiyuan 030051, China
}

\begin{abstract}
A novel preparing method of polymer-coated molybdenum powder was presented. A type of multi-component polymer-coated molybdenum powder was chosen for selective laser sintering. The effect of the process parameters on the part's characteristics is investigated. Based on our study for dynamic laser sintering process of polymer-coated molybdenum powder, its laser sintering mechanism was reported as follows: at the early stage of laser sintering, the viscous flow is the major mechanism; during the laser sintering, the melting/solidification is the major mechanism. Furthermore, a model corresponding to the mechanism was discussed schematically, which could be used to explain the material migrating mode during laser sintering process. The laser sintering experiments of polymer-coated Mo powder was conducted in the self-developed laser sintering machine, and optimized parameters have been acquired. At last, the post treatment process of laser sintered parts has been developed and refractory metal parts of $\mathrm{Mo} / \mathrm{Cu}$ composites are gained. The post treatment process includes debinding, high temperature sintering and melting infiltration, which is sintering framework-Mo by high temperature combining with $\mathrm{Cu}$ impregnation method. It is found that the mechanical properties of parts have been greatly improved after the post treatment process.
\end{abstract}

Keywords: Polymer-coated Mo powder, selective laser sintering, sintering mechanism, post treatment.

\section{INTRODUCTION}

Nowadays, Consolidation of loose powder by local laser heating is becoming a promising manufacturing technique because of the easy control over both powder deposition and laser radiation. Selective laser sintering/melting (SLS/SLM) technology makes it possible to create fully functional parts directly from metals, ceramics, plastics without using any intermediate binders or any additional processing steps after the laser sintering operation [1,2]. Therefore, SLS/SLM technologies are widely used in various industries, medicine and research offering a range of advantages compared to conventional manufacturing techniques: shorter time to market, use of inexpensive materials, higher production rate, versatility, high part accuracy, ability to produce more functionality in the parts with unique design and intrinsic engineered features [3].

SLS process begins with a completely defined CAD model of the part to be made. Divided into cross-sections by a special software, the model is then directly involved in the process. The essential operation is the laser beam scanning over the surface of a thin powder layer previously deposited on a substrate. The forming process goes along the scanning direction of the laser beam. Each cross-section (layer) of the part is sequentially filled with elongated lines (vectors) of molten powder. The quality of a part produced by this technology depends strongly on the quality of each single vector and each single layer. The powder layer thickness, the energy density and the diameter of the laser beam and the

*Address correspondence to this author at the School of Material Science and Engineering, North University of China, Taiyuan 030051, China; Tel: +86-351-3557439; Fax:+86-351-3922012; E-mail: liubin3y@nuc.edu.cn scanning speed are the crucial parameters, because these parameters happen to be the most influential on the part's characteristics (porosity, hardness and mechanical properties). Powder binding mechanisms, such as melting and solid-state or liquid-phase sintering, depend on temperature, thus local temperature fields are important for process stability and quality of the fabricated objects [4-6].

Molybdenum is widely used as refractory material. SLS process can be used to produce the molybdenum parts too. In 2006, Liu et al. [7] investigated the SLS Mo/Cu composites and its post-treatment techniques. In order to improve the forming quality of laser sintered metallic parts, it is very important to investigate the laser sintering mechanism of polymer-coated metallic powder. During selective laser sintering of polymer-coated molybdenum powder, the polymer plays the role as binder, which can bind the molybdenum particle and form the prototype. Binding is caused by laser induced localized heating, and the duration of the laser beam at any powder particle is short, typically between 0.5 and $25 \mathrm{~ms}$. Therefore the thermal-induced binding reactions must be kinetically rapid. For laser sintering of plastics, two mechanisms are put forward: viscous flow when the powder has appropriate temperaturedependent viscosity, and melting, as reported by Scherer [8] and Gusarov et al. [9] However, the study on laser sintering mechanism of polymer-coated metallic powder was seldom reported, especially for the polymer-coated molybdenum powder [10-12].

Therefore, the objective of this work is to investigate the influence of the process parameters on the part's characteristics. At the same time, a novel preparing method of polymer-coated molybdenum powder was presented. Based on our study for dynamic laser sintering process of 
polymer-coated molybdenum powder, its laser sintering mechanism was reported, which could be used to explain the material migrating mode during laser sintering process.

In this paper, selective laser sintering rapid prototyping technique of polymer-coated Mo powder was introduced, which can be used to manufacturing refractory metal parts. At last, the post treatment process of laser sintered prototyping parts has been developed and refractory metal parts of $\mathrm{Mo} / \mathrm{Cu}$ composites are gained by SLS technique.

\section{EXPERIMENTS}

\subsection{Preparation of Polymer-Coated Molybdenum Powder}

The fabricating process of polymer-coated molybdenum powder included:

1) Reductive Mo powder (purity of $99.9 \%$, maximum particle size of $5 \mu \mathrm{m}$ ) was used, the liquid polymer (3wt. \%) was added, and the mixer was mixed in the high-speed mixing machine. The block materials were prepared.

2) The blocks were dried and broken into little pieces in a crucial point machine. The polymer-coated metal powder was produced after sieving. The maximum particle diameter was $71 \mu \mathrm{m}$

Fig. (1) showed the preparing process of polymer-coated molybdenum powder.

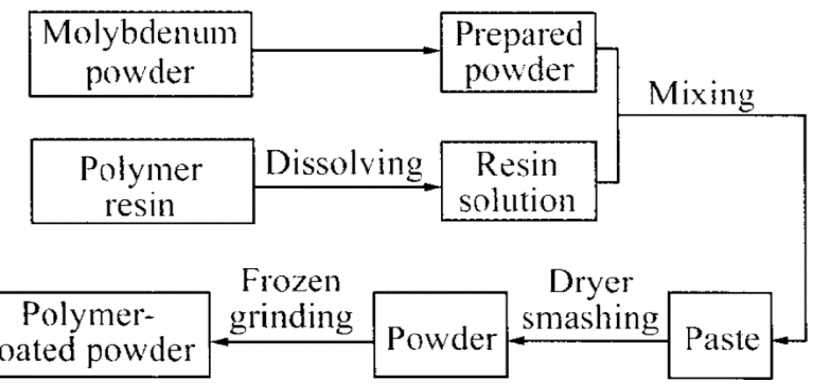

Fig. (1). Schematic diagram of Polymer-coated Mo powder preparing process.

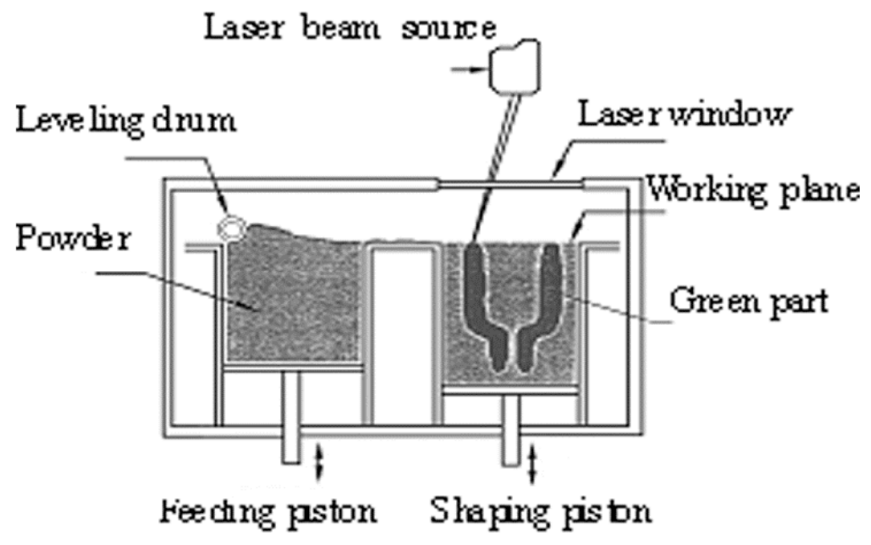

Fig. (2). Schematic diagram of SLS apparatus.

\subsection{Experimental Methods}

When the laser beam selectively scans the powder layer controlled by computer, the scanning powder is sintered. After one layer is scanned, the next powder layer is paved by leveling drum and sintered together with last layer. There are powder materials in not scanning section. At last, a green part is prototyped with cleaning surplus powder. Fig. (2) is the illustrative diagram of SLS prototyping schematic process.

\subsection{Post-Treatment Process}

SLS samples(green parts) of polymer-coated Mo powder are debinded and presintered in vacuum electric furnace. Polymer component can be eliminated through debinding process at $800 \mathrm{~K}$ for 2 hours. Then the samples are sintered at $1450 \mathrm{~K}$ and $1500 \mathrm{~K}$ for a hour, which presintering process works.

Mo framework samples have been obtained from presintered ones at $2200 \mathrm{~K}$ high temperature sintering and strengthening in hydrogen protecting furnace. One of the most effective ways that can strengthen material mechanics properties is lessening holes. Melting impregnation technique is that lower melting point metal can be infiltrated to framework holes.

Material selection of melting infiltration is $\mathrm{Cu}$, and consistency condition of $\mathrm{Cu}$ and $\mathrm{Mo}$ is considered firstly. Electrolysis pure $\mathrm{Cu}$ and framework-Mo part are placed in graphite crucible. Heating temperature is $1470 \mathrm{~K}$ in argon atmosphere, and 2 hours heat preservation is needed.

\section{RESULTS AND DISCUSSION}

\subsection{The Effect of Preheating Temperature on the Sintering Parts}

Because every layer is not cooled at the same time during the sintering process, layers and it's inner present the internal stress which can result in the warpage of sintering parts. Generally, the preheating of the material can reduce the internal stress, increase the intension and density of sintering parts, economize laser power and enhance the scanning velocity. It was proved by the experiments, non-preheating or asymmetry preheating can increase prototyping times and decrease properties and precision of sintering parts. When the materials are preheated, the density and thermal resistance increase, it is easier to form layer adhesion for the reducing internal stress. However, the preheating temperature is decided by the materials, higher preheating temperature may harden or carbonized the powder layer.

\subsection{The Effect of Scanning Speed and Laser Power on the Sintering Density and Depth}

Figs. (3-6) show the effect of scanning speed and laser power on the sintering density and depth. it can be seen that, given laser power constant, the sintering density and depth decrease with the increasing of scanning velocity; Given the scanning velocity constant, the sintering density and depth increase with the increasing of laser power. Under low scanning velocity and high laser power, the sintering depth increases. However, laser power must match the scanning velocity, higher laser power and lower scanning velocity may result in the big warpage.

\subsection{The Effect of the Powder Layer Thickness on the Sintering Density}

Fig. (7) shows the effect of the powder layer thickness on the sintered relative density. It can be seen that the sintering density decrease with the increasing of the powder layer 
thickness. While other parameters were invariable, the small powder layer thickness can obtain good layer juncture. However, the powder layer thickness is too small to spreading the powder.

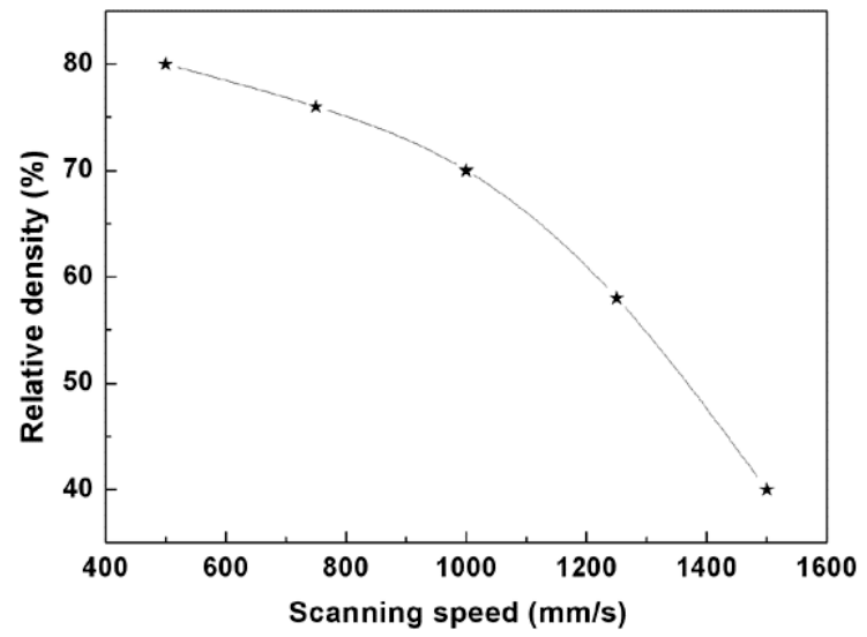

Fig. (3). The effect of the scanning speed on the relative density.

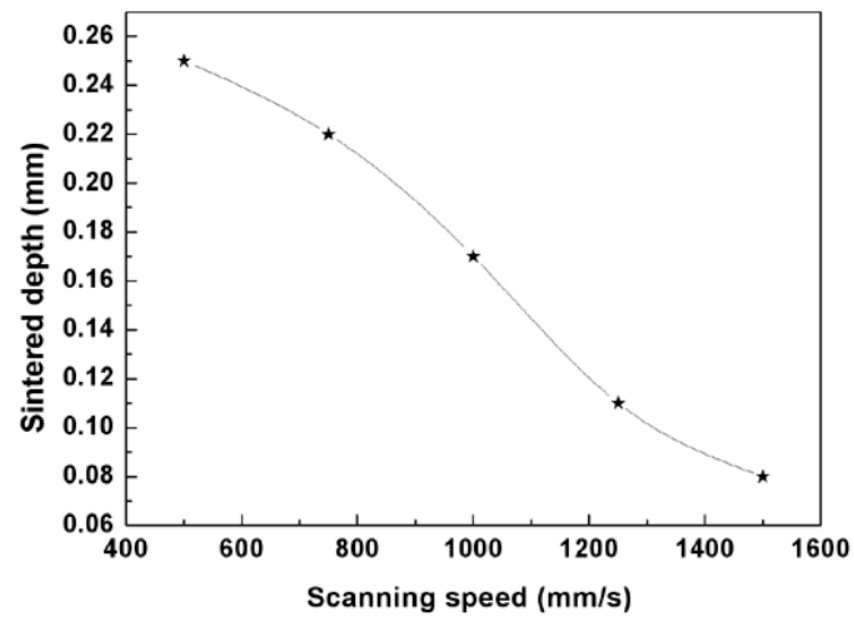

Fig. (4). The effect of the scanning speed on the sintering depth.

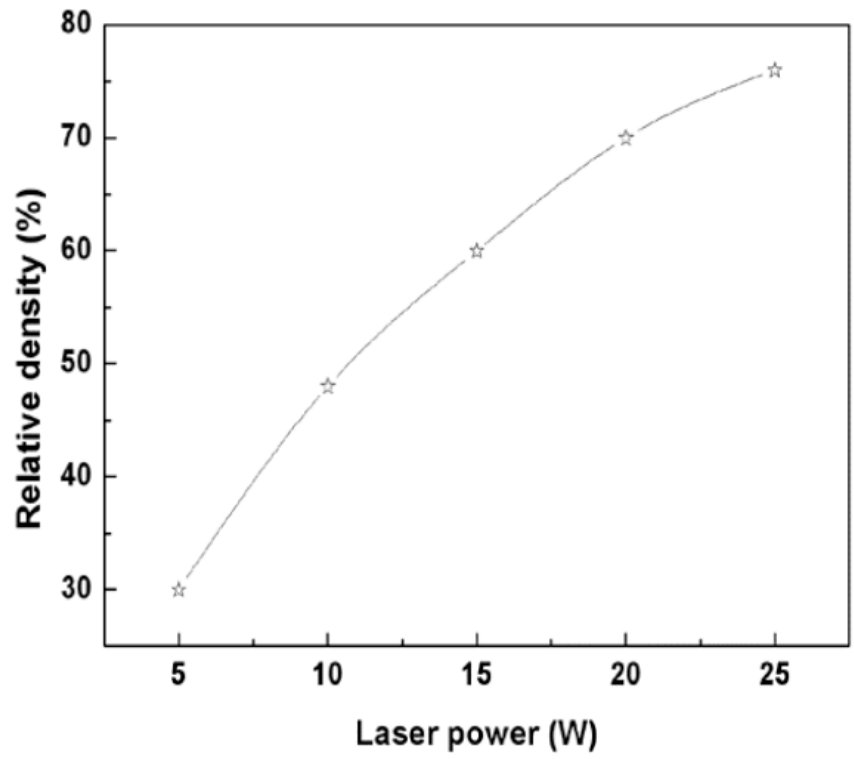

Fig. (5). The effect of the laser power on the relative density.

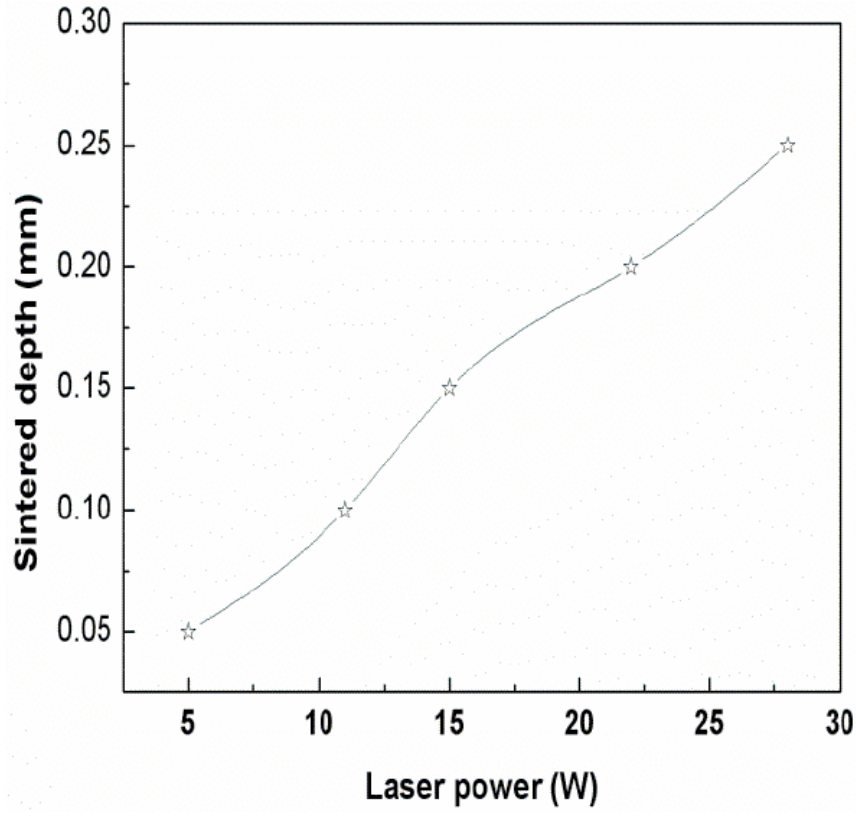

Fig. (6). The effect of the laser power on the sintered depth.

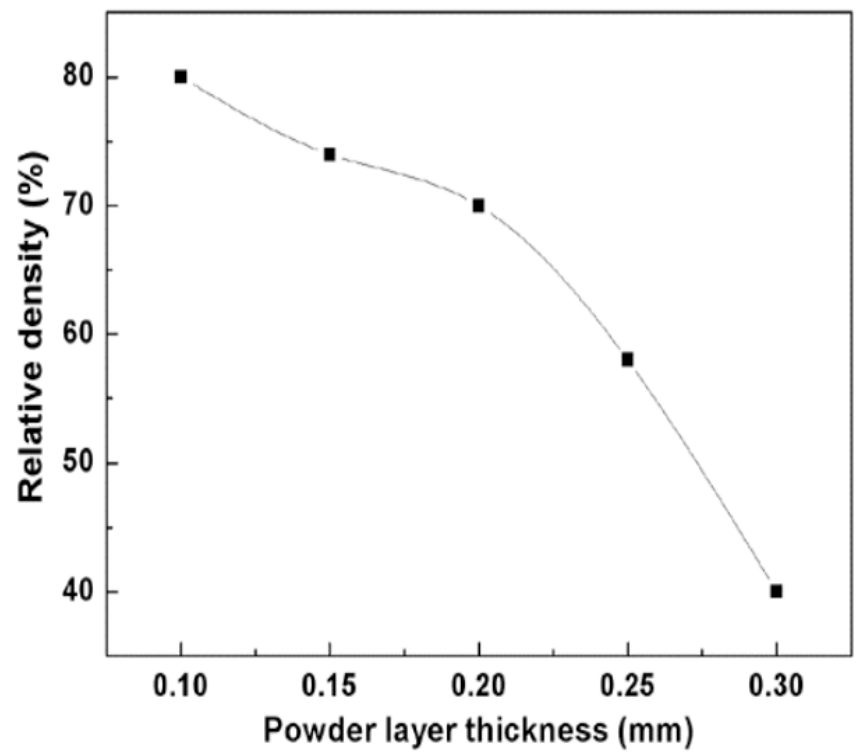

Fig. (7). The effect of the powder layer thickness on the sintered relative density.

In a word, the best sintering parameters of $\mathrm{Mo} / \mathrm{Cu}$ composites are as follows: the laser power $24 \mathrm{~W}$, the preheating temperature $60^{\circ} \mathrm{C}$, the scanning velocity $1000 \mathrm{~mm} / \mathrm{s}$, the powder bed depth $0.15 \mathrm{~mm}$.

\subsection{The Formation Mechanism of Laser Sintering}

Six basic sintering metal powder mechanisms were presented as viscous flow, plastic flow, vaporization and solidification, volume diffusion, surface diffusion as well as grain boundary diffusion [12]. Obviously, the above mechanisms cannot meet the situation of polymer-coated molybdenum sintering process.

Based on the study for dynamic laser sintering process of polymer-coated molybdenum powder, the mechanisms are presented, which can be used to explain the material 
migrating mode during laser sintering process of polymercoated molybdenum powder.

At the early stage of laser sintering, when the sintering temperature is above $100^{\circ} \mathrm{C}$, corresponding to the melting point of polycrestyne wax, the laser beam heats the powder bed locally, inducing melting of the low melting point polymer (wax) only. Wax on the surface of big particles (particles agglomerated by small polymer-coated ceramic particles in fact) melt completely, while the melting of the polymer inside the big particle is limited, which appears semimelting or solid state, as shown in Fig. (8). In this period, the viscous flow is the major mechanism.

(2) When the temperature is above $160^{\circ} \mathrm{C}$, corresponding to the melting point of PA12, PA12 melts and molten polymer amount inside the big particle increases, with fluidity getting better. The molten polymer wets the little metal particles inside big particle and fills in the pores among big particles. The sintering mechanism can be described as melting/solidification during this stage, as shown in Fig. (9). Therefore, it can be seen that the heat temperature is a crucial factor during SLS.

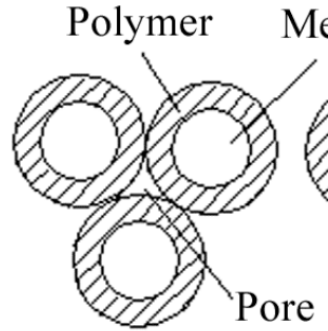

(a)

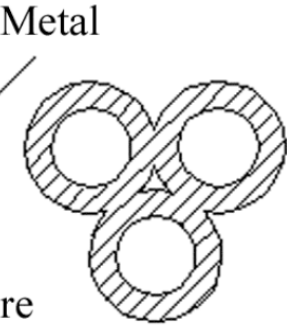

(b)

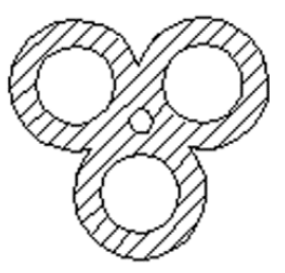

(c)
Fig. (8). The mechanism models of the early stage of laser sintering.

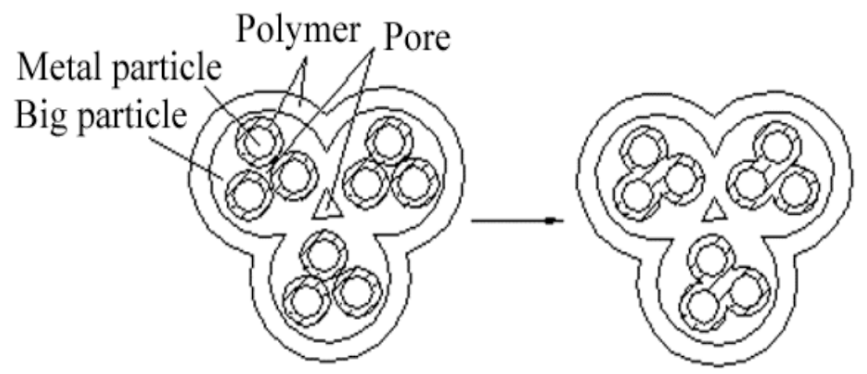

Fig. (9). The melting/solidification models of laser sintering process.

\subsection{Mo/Cu Composites}

Based on high temperature sintering theory analyses of $\mathrm{Mo} / \mathrm{Cu}$ material and large quantities of sintering experiments, the post-treatment process of prototyping green parts has been developed, which is sintering Mo framework by high temperature combining with $\mathrm{Cu}$ impregnation technique.

The quantities and size of holes are reduced through presintering process. But the samples have lower strength. Micrograph of Mo samples post treated with high temperature sintering is showed in Fig. (10). Its strength has been increased largely, and density been raised. The types of contraction are powder pellets and its groups. One result is that interior jointed structure has changed, and the next result is that dimension of powder pellets changed [13]. After high temperature sintering, Mo parts have gained high strength and better integrated properties.

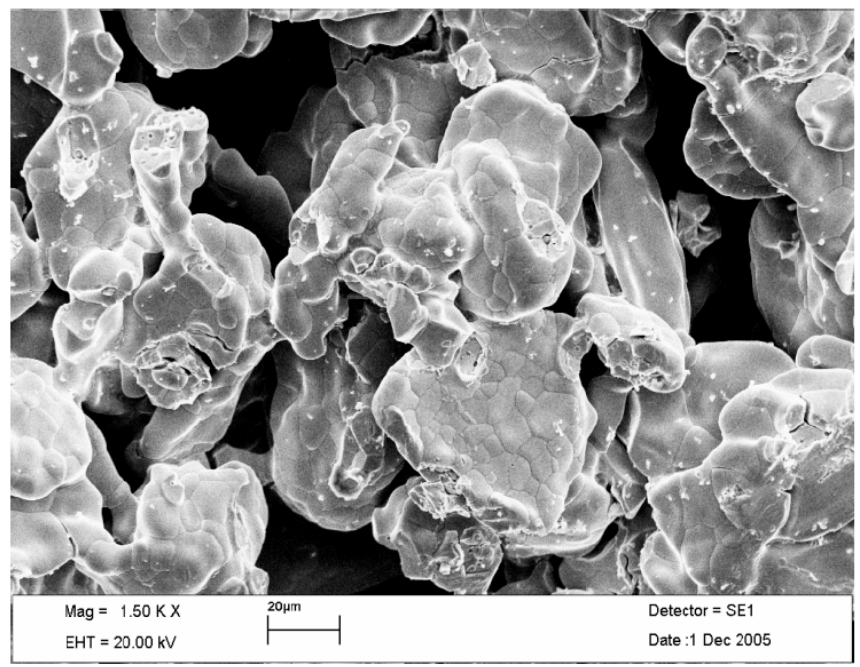

Fig. (10). SEM image of Mo framework samples by high temperature sintering.

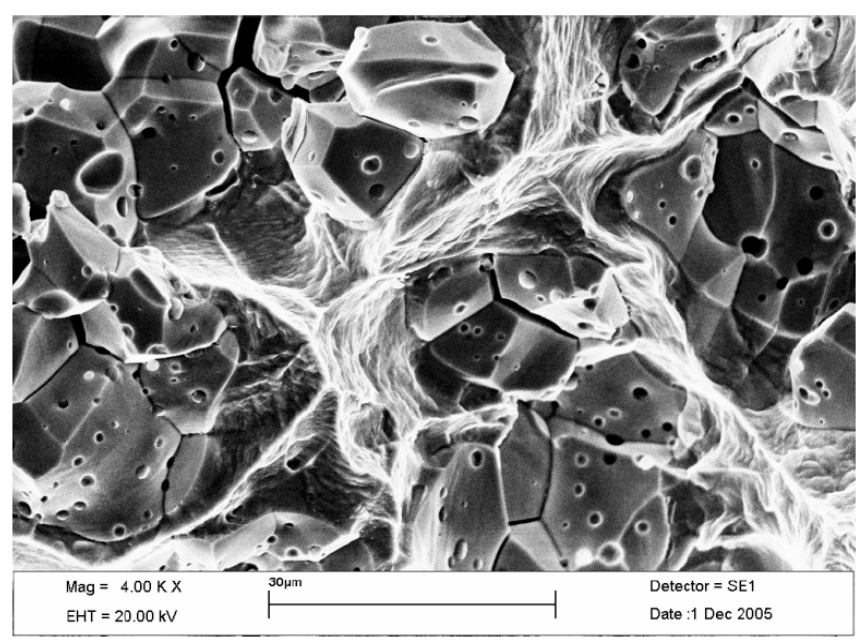

Fig. (11). SEM image of $\mathrm{Mo} / \mathrm{Cu}$ composites.

$\mathrm{Mo} / \mathrm{Cu}$ composites $(30 \mathrm{wt} \% \mathrm{Cu})$ fracture micrograph is showed as Fig. (11). SEM graph shows that $\mathrm{Mo}$ and $\mathrm{Cu}$ are close jointed together. Mo grains frequently string together. The microstructural characterization of $\mathrm{Mo} / \mathrm{Cu}$ composites is homogeneous compound structure of adhesive phase $\mathrm{Cu}$ linked with Mo grains. A concentration along grain boundaries and these phases distribute interlocked. There is little ellipsoidal Mo grains singly existed around by $\mathrm{Cu}$ phase. Between Mo grains and $\mathrm{Cu}$ zone, there is a medium changing zone, a width of $10 \sim 20 \mathrm{~nm}$.

Generally, post-treatment mechanism is Mo framework sintering of solid phase and $\mathrm{Cu}$ impregnation of melting/solidification. Melting liquid $\mathrm{Cu}$ is infiltrated into Mo framework by capillary action, and large quantities of holes are filled. Pore-solid ratio of composites is reduced mostly, and mechanics properties is heightened mostly by this alloying process. Then $\mathrm{Mo} / \mathrm{Cu}$ samples are gained which have high density and good mechanical properties. 
Many types $\mathrm{Mo} / \mathrm{Cu}$ composites of $\mathrm{Cu}$ content are able to achieved by controlling pore-solid ratio of Mo framework. After measuring and calculating, density ratio of the material is reached to $92 \%$, and mechanical properties are good enough for some using demands. For example, semi-finished products of $\mathrm{Mo} / \mathrm{Cu}$ composites are cut and grinded on local surface. Then regular products or samples of $\mathrm{Mo} / \mathrm{Cu}$ composites with precise structure and lower surface roughness would be obtained at last.

\section{CONCLUSIONS}

(1) According to the results of process experiment, the best sintering parameters of $\mathrm{Mo} / \mathrm{Cu}$ composites are as follows: the laser power $24 \mathrm{~W}$, the preheating temperature $60^{\circ} \mathrm{C}$, the scanning velocity $1000 \mathrm{~mm} / \mathrm{s}$, the powder bed depth $0.15 \mathrm{~mm}$.

Based on our study for dynamic laser sintering process of polymer-coated molybdenum powder, its laser sintering mechanism was reported as follows: at the early stage of laser sintering, the viscous flow is the major mechanism; during the laser sintering, the melting/solidification is the major mechanism. Furthermore, a model corresponding to the mechanism was discussed schematically.

(3) The post-treatment process is that Mo framework high temperature sintering combined with $\mathrm{Cu}$ impregnation technique. The microstructural evolution of post-treatment samples was investigated by SEM. Its mechanism is Mo framework sintering of solid phase and $\mathrm{Cu}$ impregnation of melting/ solidification. Mechanical and thermal properties of $\mathrm{Mo} / \mathrm{Cu}$ composites have been tested, whose density ratio is $92 \%$.

(4) Many types $\mathrm{Mo} / \mathrm{Cu}$ composites of $\mathrm{Cu}$ content are able to achieved by controlling pore-solid ratio of Mo framework. The technique can be applied to manufacturing fire-resisting parts of weaponry and aerospace arms, electronics industry conductance and cooling elements, which is owing to its excellent strength, plasticity and machinability, as well as electrical and thermal conductivities.

\section{REFERENCES}

[1] Terry T. Wohlers. Wohlers report 2007: state of the industry : annual worldwide progress report 2007; 5: 220-7.

[2] Kumar S, Kruth JP. Composites by rapid prototyping technology. Materials and Design 2010;31: 850-6.

[3] Terry Wohlers et al. Rapid Prototyping, Tooling \& Manufacturing State of the Industry; Annual Worldwide Progress Report 2005; 11(3): 160-6.

[4] Simchi A, Pohl H. Direct laser sintering of iron-graphite powder mixture. Mater Sci Eng A 2004; 383: 191-200.

[5] Kruth JP, Mercelis P, Van Vaerenbergh J. Binding mechanisms in selective laser sintering and selective laser melting. Rapid Prototyping J 2005; 11: 26-36.

[6] Yadroitsev I, Thivillon L, Bertrand Ph, Smurov I. Strategy of manufacturing components with designed internal structure by selective laser melting of metallic powder. Appl Surface Sci 2007; 254: 980-3.

[7] Liu B, Cheng J, Bai PK. Preparation of $\mathrm{Mo} / \mathrm{Cu}$ composites using SLS and its post-treatment techniques. Trans Nonferrous Met Soc China 2006; 16(S2): 255-9.

[8] Scherer GW. Viscous Sintering under a Uniaxial Load. J Am Ceramic Soc 1986; 69(9): C206-7.

[9] Gusarov AV, Laoui T, Froyen L. Contact thermal conductivity of a powder bed in selective laser sintering. Int J Heat Mass Transfer 2003; 46: 1103-9.

[10] Tolochko NK, Mozzharov SE, Yadroitsev IA. Selective laser sintering and cladding of single -component metal powders. Rapid Prototyping J 2004; 10: 88-97.

[11] Childs THG, Hauser G, Badrossamay M. Selective laser sintering (melting) of stainless and tool steel powders: experiments and modeling. J Eng Manufact 2005; 219: 339-57.

[12] P.K. Bai, J. Cheng, Study on selective laser sintering of polymercoated metal powder. Proc Second Int Conference Rapid Prototyping Manufact 2002; 449-53.

[13] Shen YF, Gu DD. Microstructural evolution during direct laser sintering of multi-component Cu-based metal powder. Trans Nonferrous Met Soc China 2005; 15: 1309-14.

This is an open access article licensed under the terms of the Creative Commons Attribution Non-Commercial License (http://creativecommons.org/licenses/ by$\mathrm{nc} / 3.0 /$ ) which permits unrestricted, non-commercial use, distribution and reproduction in any medium, provided the work is properly cited. 